\title{
Cytoplasmic translocation of high-mobility group box-1 protein is induced by diabetes and high glucose in retinal pericytes
}

\author{
JUNGHYUN KIM, CHAN-SIK KIM, EUNJIN SOHN and JIN SOOK KIM
}

Korean Medicine Convergence Research Division, Korea Institute of Oriental Medicine, Daejeon 34054, Republic of Korea

Received August 4, 2015; Accepted July 18, 2016

DOI: $10.3892 / \mathrm{mmr} .2016 .5702$

\begin{abstract}
The aim of the present study was to assess the involvement of the high-mobility group box-1 (HMGB1) protein, receptor for advanced glycation end products (RAGE) and nuclear factor (NF)- $\kappa \mathrm{B}$ signaling pathway in the development of diabetic retinopathy. Rat primary retinal pericytes were exposed to $25 \mathrm{mmol} / \mathrm{l} \mathrm{D}$-glucose for $48 \mathrm{~h}$. Diabetic retinal vessels were prepared from streptozotocin-induced diabetic rats 12 weeks following the induction of diabetes. The expression of HMGB1 was detected using immunofluorescence staining. The expression of RAGE and the activity of NF- $\mathrm{NB}$ were analyzed using western blot and electrophoretic mobility shift assays, respectively. The results showed that HMGB1 was translocated to the cytoplasm of the high glucose-treated pericytes and diabetic retinal pericytes, whereas, in the control cells and the normal retinas, HMGB1 was expressed in the cell nuclei only. The expression of RAGE, a potential receptor for HMGB1, and the activity of $\mathrm{NF}-\kappa \mathrm{B}$ were also increased in the high glucose-treated pericytes, compared with the normal control cells. In addition, high glucose increased the binding of $N F-\kappa B$ to the RAGE promoter. These findings suggested that the cytoplasmic translocation of HMGB1 may be caused by diabetes and high glucose in retinal pericytes, and that the pathogenic role of HMGB1 may be dependent on the expression of RAGE and activation of NF- $\mathrm{\kappa B}$.
\end{abstract}

\section{Introduction}

Diabetic retinopathy, the leading cause of blindness worldwide, is characterized by early dysfunction of the retinal microvasculature. Metabolic alterations resulting from hyperglycemia are considered to be the cause of diabetic retinopathy (1). It has been reported that inflammatory processes are also important

Correspondence to: Dr Jin Sook Kim, Korean Medicine Convergence Research Division, Korea Institute of Oriental Medicine, 1672 Yuseongdaero, Yuseong-gu, Daejeon 34054, Republic of Korea

E-mail: jskim@kiom.re.kr

Key words: diabetic retinopathy, high-mobility group box-1, retinal pericyte in the pathophysiology of diabetic retinopathy (2). Leukocyte infiltration and the enhanced expression of chemokines, adhesion molecules, growth factors and nuclear factors have been observed in diabetic retinal tissues (3).

The high-mobility group box-1 (HMGB1) protein was originally described as a nuclear DNA-binding protein (4), which facilitates gene transcription by stabilizing nucleosome formation (5). HMGB1 can also be released extracellularly, and acts as a pro-inflammatory cytokine or as an alarm signal for tissue damage (6). HMGB1 is released from cells through passive release or by active secretion. Passive release occurs as a result of cellular necrosis in the majority of eukaryotic cells $(7,8)$. Active secretion from activated macrophages and monocytes occurs in response to inflammatory stimuli, including lipopolysaccharide and tumor necrosis factor (TNF)- $\alpha(9,10)$, and can trigger a potent inflammatory response leading to severe tissue injury $(11,12)$. Extracellular HMGB1 is also involved in the progression of several inflammatory conditions, including septic shock, rheumatoid arthritis and atherosclerosis (13-16). Previously, diabetes has been shown to be associated with an increase in the expression of HMGB1 in aortic endothelial cells in vivo, and HMGB1 has been suggested as a causative factor in diabetic tissue damage (17). Inflammation has been recognized as being important in the pathogenesis of diabetic retinopathy, and anti-inflammatory agents can be beneficial in diabetic retinopathy (18), for example dexamethasone suppressed upregulation of intercellular adhesion molecule 1, leukostasis, and prevented retinal vascular leakage in a rat model of diabetic retinopathy (19). In addition, Jonas and Söfker (20) reported that an intravitreal injection of triamcinoloe acetonide efficiently improved visual acuity in diabetic patients with macular edema. However, the role of HMGB1 in diabetic retinopathy remains to be fully elucidated. Therefore, the present study aimed to investigate the pathogenic function of HMGB1, focusing on its role in diabetic retinopathy, using primary rat retinal pericytes and streptozotocin (STZ)-induced diabetic rats. The involvement of the receptor for advanced glycation end products (RAGE) and the nuclear factor (NF)- $\mathrm{B}$ signaling pathway were also examined.

\section{Materials and methods}

Primary rat retinal pericyte cell culture. The primary retinal pericytes were isolated from the retinal microvessels of Sprague-Dawley rats (Orient Bio, Inc., Seoul, Korea) using a 
modified version of a previously published method(21-24). After one week acclimation period, eight eyes from four rats were enucleated under deep anesthesia, following intraperitoneal injection of pentobarbital sodium $(30 \mathrm{mg} / \mathrm{kg}$ body weight; Hanlim Pharmaceuticals Co., Ltd., Seoul, Korea). Animals were then sacrificed with an overdose of pentobarbital sodium (200 mg/kg body weight; Hanlim Pharmaceuticals Co., Ltd.). The retinas were separated from the eyes, homogenized using a Teflon-glass homogenizer (Wheaton, Millville, NJ, USA) and filtered though a $70-\mu \mathrm{m}$ nylon mesh (BD Biosciences, San Diego, CA, USA). The remaining retentate was digested in $0.066 \%$ collagenase/dipase (Roche, Mannheim, Germany) in Dulbecco's phosphate-buffered saline for $1 \mathrm{~h}$ at $37^{\circ} \mathrm{C}$. The cellular digests were then filtered through a $40-\mu \mathrm{m}$ nylon mesh. Purification of the rat retinal pericytes was achieved using a CELLection Pan Mouse IgG kit (Invitrogen; Thermo Fisher Scientific, Inc., Waltham, MA, USA) with a mouse anti-desmin monoclonal antibody (cat. no. MAB3430; EMD Millipore, Billerica, MA, USA), according to the manufacturer's protocol. The purified cells were maintained in Dulbecco's modified Eagle's medium containing $10 \%$ fetal bovine serum at $37^{\circ} \mathrm{C}$ in a humidified atmosphere of a $5 \% \mathrm{CO}_{2}$ incubator. The pericytes were identified by their inability to uptake rhodamine-conjugated, acetylated low-density lipoprotein, as described by Cacicedo et al (25). The cell cultures in the present study contained no cells, which reacted with polyclonal rabbit anti-human antibodies to the endothelial cell marker, von Willebrand factor (cat. no. A0082; Dako, Carpinteria, CA, USA). Cells between passages 3 and 5 were used in the present study. The cells $\left(5 \times 10^{4}\right)$ were plated onto appropriate culture dishes and used for experiments upon reaching $80 \%$ confluence. Standard culture medium was replaced with fresh serum-free medium $16 \mathrm{~h}$ prior to the experiments at $37^{\circ} \mathrm{C}$. To examine the effects of high glucose, the medium of confluent pericyte cultures was supplemented with $25 \mathrm{mmol} / \mathrm{l} \mathrm{D}$-glucose for $48 \mathrm{~h}$. Galactose or mannitol were used as a control.

Immunofluorescence staining. The immunofluorescence staining was performed on the cultured pericytes. The antibody used was monoclonal rabbit anti-HMGB1 (2639-1; Epitomics, San Fransisco, CA, USA). For the detection of HMGB1, the cells were incubated with a fluorescein isothiocyanate (FITC)-conjugated goat anti-rabbit antibody (Santa Cruz Biotechnology, Santa Cruz, CA, USA). The fluorescence signals were observed under a fluorescence microscope (Olympus Corporation, Tokyo, Japan) and analyzed using ImageJ software (National Institutes of Health, Bethesda, MD, USA).

Western blot analysis. The nuclear and cytoplasmic extracts were prepared according to a method described by Schreiber et al (26). Briefly, $1 \times 10^{7}$ cells were resuspended in $400 \mathrm{ml}$ of cold buffer A, containing $10 \mathrm{mM}$ HEPES (pH 7.9), $10 \mathrm{mM} \mathrm{KCl}, 0.1 \mathrm{mM}$ EDTA, $0.1 \mathrm{mM}$ EGTA, $1 \mathrm{mM}$ DTT and $0.5 \mathrm{mM}$ PMSF. The cells were allowed to swell on ice for $15 \mathrm{~min}$, following which $25 \mathrm{ml}$ of a $10 \%$ solution of was added, and the tube was vigorously vortexed for $10 \mathrm{sec}$. The homogenate was centrifuged at $12,000 \mathrm{x}$ g for $30 \mathrm{sec}$ in a microfuge at $4^{\circ} \mathrm{C}$, with the supernatant containing the cytoplasm. The nuclear pellet was resuspended in $50 \mathrm{ml}$ of ice-cold buffer $\mathrm{B}$, containing $20 \mathrm{mM}$ HEPES (pH 7.9), $0.4 \mathrm{M} \mathrm{NaCl}, 1$ mM EDTA,
$1 \mathrm{mM}$ EGTA, $1 \mathrm{mM}$ DTT and $1 \mathrm{mM}$ PMSF, and the tube was vigorously rocked at $4^{\circ} \mathrm{C}$ for $15 \mathrm{~min}$ on a shaking platform. The nuclear extract was centrifuged at $12,000 \mathrm{x}$ g for $5 \mathrm{~min}$ in a microfuge at $4^{\circ} \mathrm{C}$, and the supernatant was frozen. The protein content of the fractions were determined using the Bradford assay method (27). The nuclear and cytoplasmic extracts $(20 \mu \mathrm{g})$ were then separated by $12 \%$ SDS-polyacrylamide gel electrophoresis and transferred onto nitrocellulose membranes (Bio-Rad Laboratories, Inc., Hercules, CA, USA). The membranes were probed with rabbit anti-HMGB1 (Epitomics), polyclonal rabbit anti-RAGE antibody (ab3611; Abcam, Cambridge, MA, USA), monoclonal mouse anti- $\beta$-actin antibody (A5441; Sigma-Aldrich; Merck Millipore, Darmstadt, Germany), monoclonal mouse anti- $\beta$-tubulin antibody (sc-5274; Santa Cruz Biotechnology, Inc.) and mouse monoclonal anti-proliferating cellular nuclear antigen (PCNA) antibody (50-171-599; Upstate Biotechnology, Inc., Lake Placid, NY, USA), and the immune complexes were then visualized using an enhanced chemiluminescence detection system (Amersham Biosciences, Uppsala, Sweden). The protein expression levels were determined by analyzing the signals captured on the nitrocellulose membranes using an image analyzer (Las-3000; Fujifilm, Tokyo, Japan). Anti- $\beta$-actin, anti- $\beta$-tubulin (28) and anti-PCNA (29) served as loading controls.

Measurement of $N F-\kappa B$ activity. For the electrophoretic mobility shift assay (EMSA), the nuclear extracts were prepared using a kit, according to the manufacturer's protocol (NE-PER Nuclear and Cytoplasmic Extraction kit; Pierce Biotechnology, Inc., Rockville, IL, USA). The EMSA was performed by incubating $10 \mu \mathrm{g}$ of nuclear protein extract with IRDye 700-labeled NF- $\kappa \mathrm{B}$ oligonucleotide probe (5'-AGT TGA GGG GAC TTT CCC AGG C-3'; LI-COR Biosciences, Lincoln, NE, USA) or an unlabelled probe for cold competition. The EMSA gels were analyzed, and images were captured and quantified using the LI-COR Odyssey infrared laser imaging system (LI-COR Biosciecnes).

Chromatin immunoprecipitation (ChIP) assay. A ChIP assay was performed to analyze the in vitro interactions of $\mathrm{NF}-\kappa \mathrm{B}$ with its cognate $c i s$-acting element in the RAGE promoter. This assay was performed using a ChIP assay kit (Upstate Biotechnology, Inc.), according to the manufacturer's protocol. The soluble chromatin was prepared the from retinal pericytes. Chromatin was mechanically sheared by sonication to yield fragments with a mean size of $300 \mathrm{bp}$. Prior to immunoprecipitation, chromatin (10 $\mu \mathrm{g}$ DNA/assay) was pretreated with protein A/G agarose beads (Santa-Cruz Biotechnology, Inc.) for $1 \mathrm{~h}$. The chromatin was immunoprecipitated with a polyclonal rabbit anti-p65 NF- $\kappa$ B antibody (sc-372; $2 \mu \mathrm{g}$ each; Santa Cruz Biotechnology, Inc.). Normal rabbit IgG was used as negative antibody control and DNA from the input (20-40 $\mu \mathrm{g}$ protein-DNA complex) as an internal control. Antibody-bound chromatin was eluted by heating at $95^{\circ} \mathrm{C}$ for $30 \mathrm{~min}$. The DNA was then purified using the QIAquick PCR Purification kit (Qiagen GmbH, Hilden, Germany). The input DNA and the DNA from Chip samples were used as a template for polymerase chain reaction amplification using primer sets for the rat RAGE promoter regions containing the $\mathrm{NF}-\kappa \mathrm{B}$ response element. The sequences of the primers were as follows: Forward 5'-CCCGGCCCTGACTAA 
A
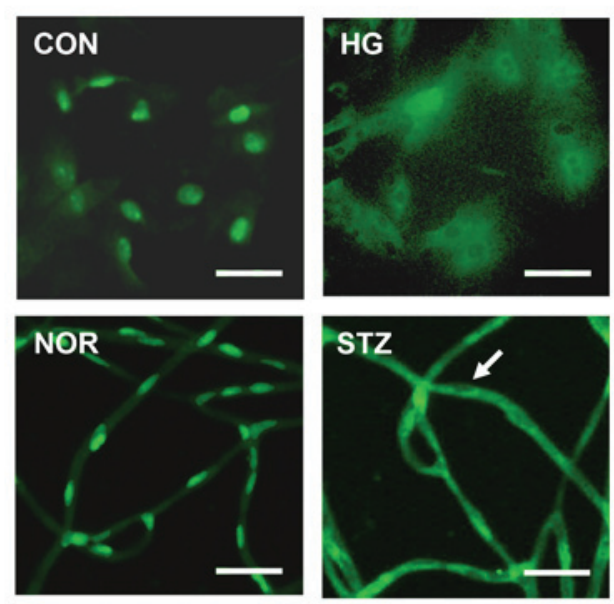

B

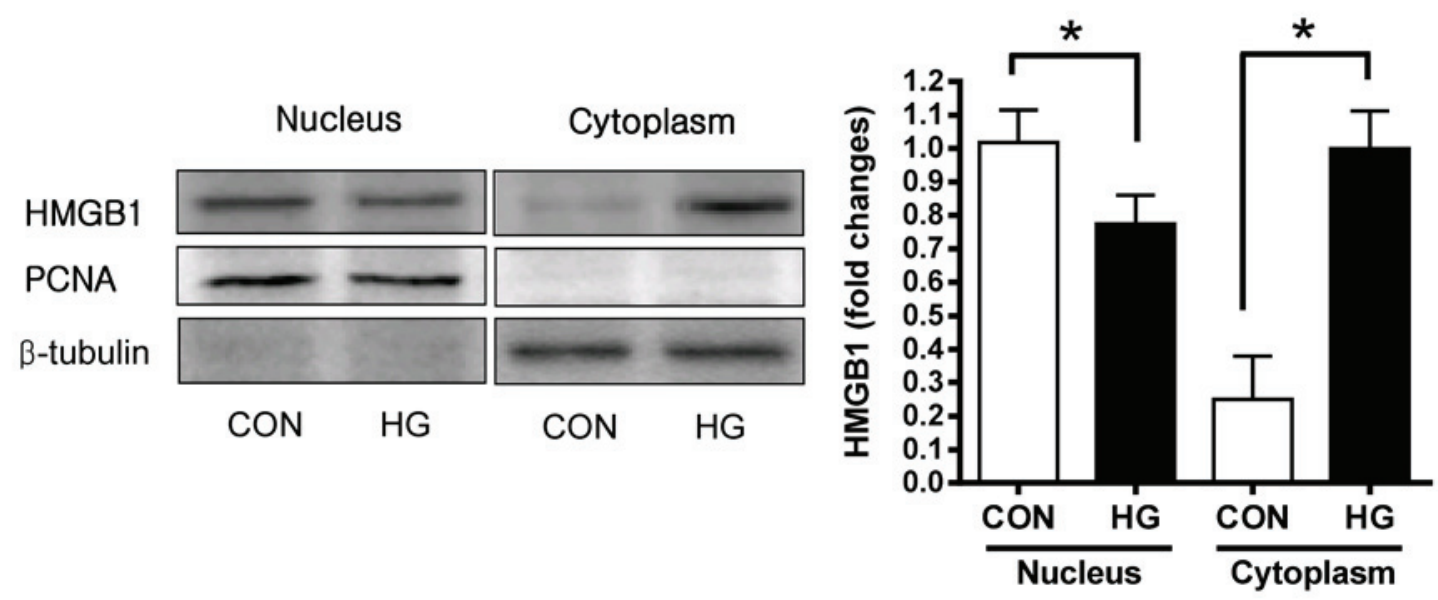

Figure 1. Cytoplasmic translocation of HMGB1 in retinal pericytes by high glucose in vitro and under diabetic conditions in vivo. (A) Immunofluorescence staining of HMGB1. (B) Western blot analysis of HMGB1 protein. Scale bar $=25 \mu \mathrm{m}$; white arrow indicates the cytoplasmic translocation of HMGB1 protein. Values in the bar graphs represent the mean \pm standard error of the mean $(n=4)$. * $<<0.05$, vs. CON. HMGB1, high-mobility group box-1; STZ, streptozotocin; CON, normal control pericytes; HG, high glucose-treated pericytes; NOR, normal rats; STZ, STZ-induced diabetic rats.

GCAGT-3' and reverse 5'-CCACGGCCTGGAACCCTTA-3'. Quantitative polymerase chain reaction (qPCR) was performed using SYBR Green PCR Master Mix and Chromo4 Multicolor Real-Time PCR Detection System (Bio-Rad Laboratories, Inc.,) with with the following cycling conditions: 2 min at $94^{\circ} \mathrm{C}$, followed by 35 cycles of $30 \mathrm{sec}$ at $94^{\circ} \mathrm{C}, 30 \mathrm{sec}$ at $58^{\circ} \mathrm{C}$ and $1 \mathrm{~min}$ at $72^{\circ} \mathrm{C}$, followed by $1 \mathrm{~min}$ at $72^{\circ} \mathrm{C}$. The copy number for each gene was determined using iQ5 Optical system software (Bio-Rad Laboratories, Inc.). The results are reported as the ratio of the immunoprecipitated DNA to the input DNA.

Animals and experimental design. Six-week-old male Sprague-Dawley rats were purchased from Orient Bio, Inc. Rats were housed under a 12-h light/12-h dark cycle at a temperature of $23 \pm 1^{\circ} \mathrm{C}$ and were provided with food and water ad libitum. After a one week acclimation period, diabetes was induced by a single injection of STZ (60 mg/kg body weight; i.p.) in the rats. Age-matched control rats (aged 7 weeks) were injected with vehicle only. At 1 week post-induction of diabetes, the blood glucose levels were measured in venous blood from the tail vein. The glucose assay used was an enzymatic assay based on glucose oxidase and peroxidase levels (Glucose B-Test; Wako, Osaka, Japan). Rats with a plasma glucose level $>300 \mathrm{mg} / \mathrm{dl}$ were considered to be diabetes-induced rats. The animals were divided into two groups: Normal rats $(n=8)$ and STZ-induced diabetic rats $(n=8)$. At 21 weeks of age, blood samples were collected from the tail vein following a 16-h fast At necropsy, the eye from each rat was enucleated under deep anesthesia, following intraperitoneal injection of pentobarbital sodium (30 mg/kg body weight; Hanlim Pharmaceuticals Co., Ltd.). Animals were then sacrificed with an overdose of pentobarbital sodium (200 mg/kg body weight; Hanlim Pharmaceuticals Co., Ltd.). All the procedures involving rats were approved by the Korea Institute of Oriental Medicine Institutional Animal Care and Use Committee (Daejeon, Korea). The blood glucose values were $6.93 \pm 0.56 \mathrm{mmol} / \mathrm{l}$ for normal rats and $22.74 \pm 3.78 \mathrm{mmol} / \mathrm{l}$ for STZ-induced diabetic rats. The body weights were $494.46 \pm 8.73 \mathrm{~g}$ (normal rats) and 229.25 $\pm 10.66 \mathrm{~g}$ (diabetic rats).

Trypsin-digested retinal vessel preparation. The eyes of the animals were enucleated and the retinas were isolated. The retinal samples were then placed in $10 \%$ formalin for 2 days. Following fixation, the retina was incubated in trypsin $(3 \%$ in sodium phosphate buffer) for $\sim 60 \mathrm{~min}$ at $37^{\circ} \mathrm{C}$. The vessel structures were isolated from the retinal cells by gentle rinsing in distilled water. The vascular specimens were then mounted 
A

B
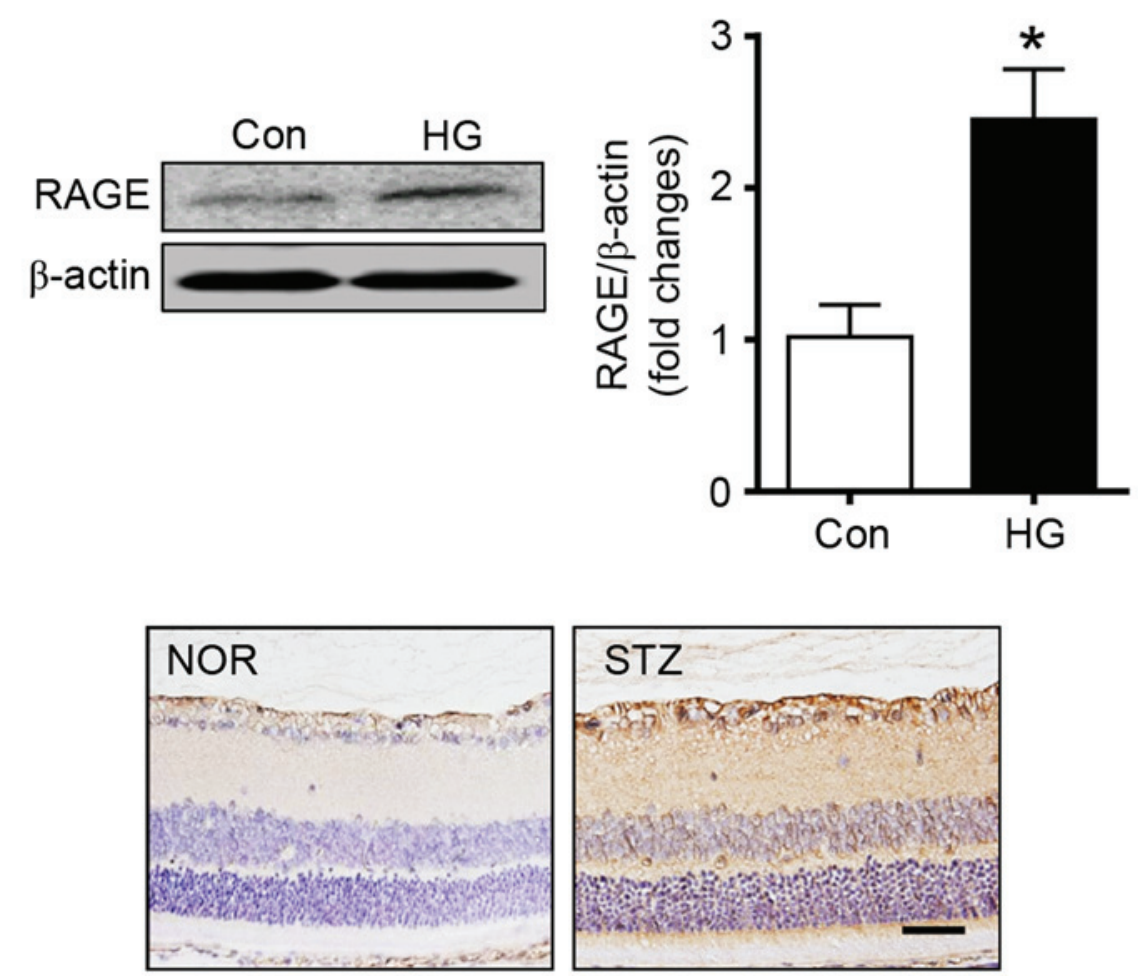

Figure 2. Expression of RAGE. (A) Western blot analysis of the protein expression of RAGE. The bands were detected and quantified using an image analyzer and RAGE protein levels were normalized to that of $\beta$-actin. Values represent the mean \pm standard error $(n=8)$. ${ }^{*} \mathrm{P}<0.01$, vs. CON. (B) Immnohistochemical staining for the expression of RAGE in the retina. Scale bar=50 $\mu \mathrm{m}$. RAGE, receptor for advanced glycation end products; CON, normal control pericytes; $\mathrm{HG}$, high glucose-treated pericytes.

on a slide. For immunofluorescence staining for HMGB1 and $\mathrm{NF}-\kappa \mathrm{B}$, the vascular specimens were incubated with rabbit anti-HMGB1 (Epitomics) and mouse anti-NF- $\kappa \mathrm{B}$ antibody (cat. no. MAB3026; Chemicon International, Inc., Temecula, CA, USA). For the detection of HMGB1, the vessels were incubated with FITC-conjugated polyclonal goat anti-rabbit antibody (sc-2012; Santa Cruz Biotechnology, Inc.). To detect NF-кB, the vessels were incubated with polyclonal FITC-conjugated goat anti-mouse IgG (sc-2010; Santa Cruz Biotechnology, Inc.) and detected using fluorescence microscopy (Olympus Corporation). For negative controls, the sections were incubated with non-immune serum instead of the primary antibodies.

Immunohistochemical staining. The retinal tissues were fixed in $10 \%$ formaldehyde and embedded in paraffin, and $4 \mu \mathrm{m}$ thick sections were prepared. The primary antibody used was rabbit anti-RAGE (Sigma-Aldrich; Merck Millipore). Following incubation in CAS blocking solution (Zymed Life Technologies, Carlsbad, CA, USA) for $30 \mathrm{~min}$, the sections were incubated in the primary antibody overnight at $4^{\circ} \mathrm{C}$ and then washed three times with phosphate-buffered saline. To detect RAGE in the retinal sections, the slides were prepared using an Envision kit (Dako), and immunoreactivity was visualized using a 3,3'-diaminobenzidine tetrahydrochloride peroxidase substrate kit (Dako).

Statistical analysis. All data are presented as the mean \pm standard error. Comparisons between the two groups were made using Student's $t$-test (two-tailed). Statistical analysis was performed using GraphPad Prism 4.0 software (GraphPad
Software, Inc., La Jolla, CA, USA). P<0.05 was considered to indicate a statistically significant difference.

\section{Results}

Expression of HMGB1 in retinal pericytes. To investigate the pathogenic functions of HMGB1 in retinal pericytes under diabetic conditions, the present study initially determined whether the protein was expressed in retinal pericytes using immunofluorescence staining. HMGB1 was detected in the nuclei and diffusely in the cytoplasm in the high glucose-treated pericytes, however, in the control, HMGB1 was expressed in the nuclei only (Fig. 1A; upper panels). Additionally, in diabetic retinal vessels, HMGB1 was expressed at a high level in the cytoplasm of the retinal pericytes (Fig. 1A; lower panels). To confirm that the HMGB1 protein had been translocated into the cytoplasm of the pericytes, the cells were subcellular fractionated, and the distribution of HMGB1 was analyzed using western blot analysis. As shown in Fig. 1B, translocation of HMGB1 from the nucleus to the cytoplasm was observed at $48 \mathrm{~h}$ following high glucose treatment. In the control pericytes, the majority of the HMGB1 was found in the nucleus, and the expression of HMGB1 was lower in the soluble cytoplasm. In the high glucose-treated pericytes, a lower level of HMGB1 was detected in the nuclear fraction and a higher level of HMGB1 was detected in the cytoplasm, compared with the control. These results suggested that HMGB1 was translocated into the cytoplasm in retinal pericytes in response to high glucose conditions in vitro and in vivo. 
$\mathbf{A}$
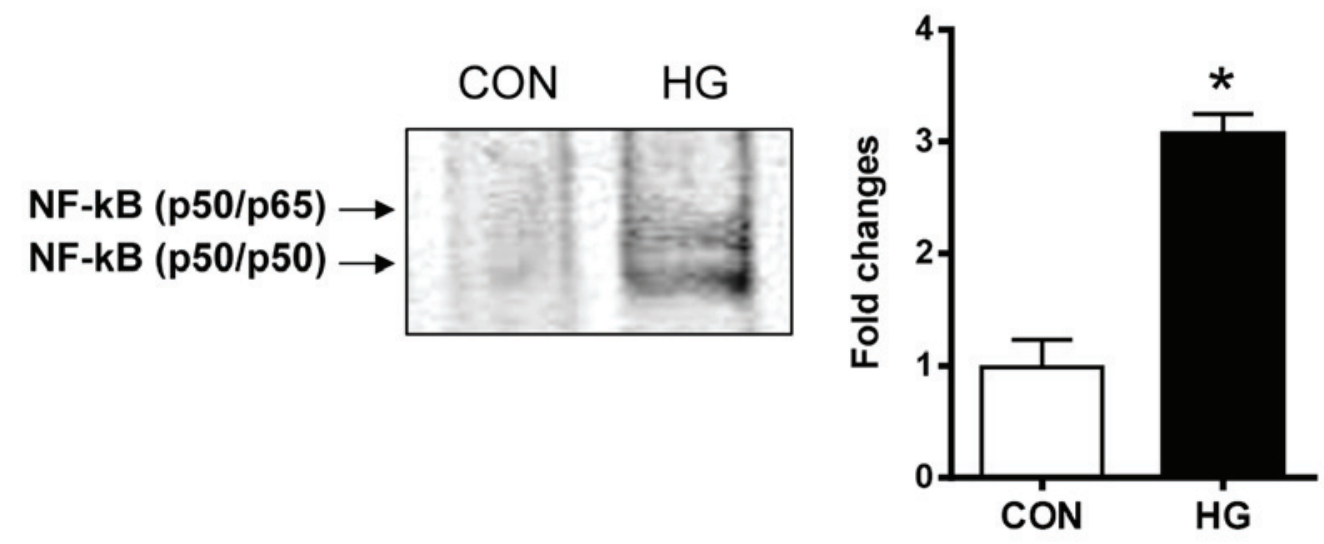

B
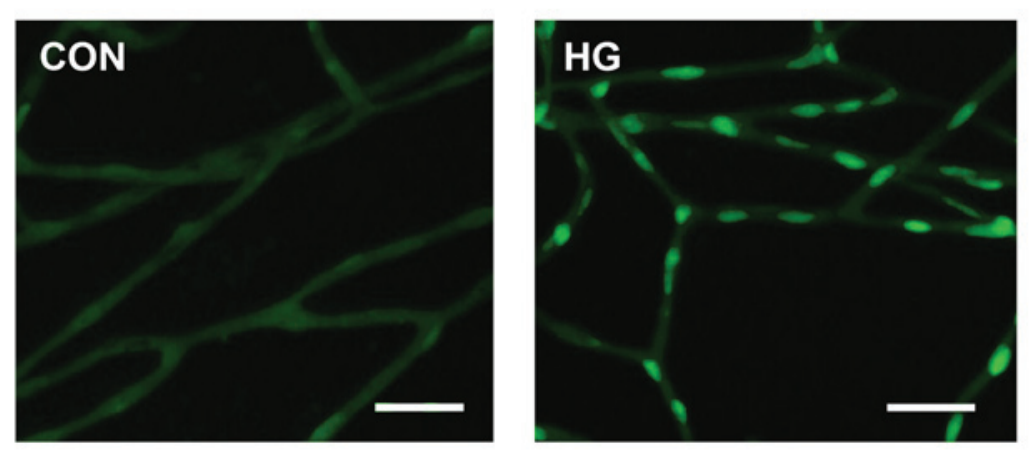

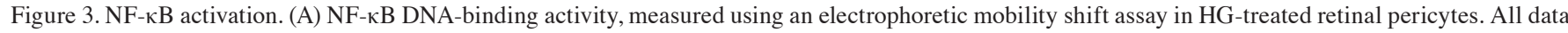
are expressed as the mean \pm standard error of the mean $(n=8) .{ }^{*} P<0.01$, vs. CON (B) Immunofluorescence staining of NF- $\kappa B$ in the retinal vessels from diabetic

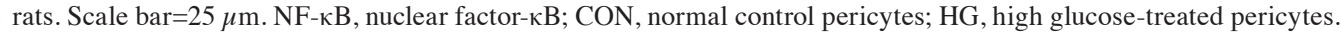

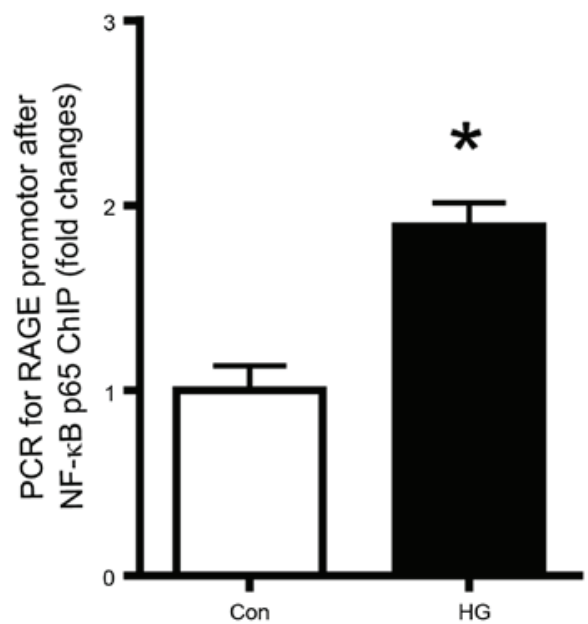

Figure 4. Effect of NF- $\mathrm{KB}$ on the expression of RAGE promoter. A ChIP assay was performed using NF- $\mathrm{BB}$ p65 antibody, and the RAGE promoter was amplified by PCR. The values in the bar graphs represent the mean \pm standard error of the mean $(n=8)$. ${ }^{*} \mathrm{P}<0.01$, vs. normal. NF- $\kappa \mathrm{B}$, nuclear factor- $\mathrm{kB}$; RAGE, receptor for advanced glycation end products; ChIP, chromatin immunoprecipitation; PCR, polymerase chain reaction; CON, normal control pericytes; HG, high glucose-treated pericytes.

Expression of RAGE. RAGE is a putative receptor for HMGB1 (30). The signaling pathways downstream of RAGE are key in the cellular responses to stress conditions, including inflammation (31). Thus, the present study measured the expression levels of RAGE in retinal pericytes. The western blot analysis showed that the protein expression of RAGE was markedly increased in the high glucose-treated pericytes, compared with the control cells (Fig. 2A). Similarly, immunohistochemical staining for RAGE showed that the expression level of RAGE was markedly higher in the diabetic rat retinas, compared with the normal rat retinas (Fig. 2B).

Activation of $N F-\kappa B$. The present study investigated whether the downstream effect of RAGE activation was associated mechanistically with the $\mathrm{NF}-\kappa \mathrm{B}$ pathway. The nuclear extracts from the retinal pericytes were analyzed for $\mathrm{NF}-\kappa \mathrm{B}$ DNA-binding activity using an EMSA. The results of the EMSA showed that the NF- $\kappa$ B DNA-binding activity was significantly increased in the high glucose-treated pericytes, compared with the control cells (Fig. 3A). In the diabetic rats, activated $\mathrm{NF}-\kappa \mathrm{B}$ was found in the nucleus of the retinal pericytes. However, in the control rats, minimal positive signals of activated $\mathrm{NF}-\kappa \mathrm{B}$ were detected (Fig. 3B). These observations indicated that diabetic conditions markedly induced $\mathrm{NF}-\kappa \mathrm{B}$ DNA-binding activity in the retinal pericytes.

Role of $N F-\kappa B$ in the mRNA expression of RAGE. To evaluate the role of $\mathrm{NF}-\kappa \mathrm{B}$ in the high glucose-induction of the mRNA expression of RAGE in the retinal pericytes, the binding of $\mathrm{NF}-\kappa \mathrm{B}$ p65 to the RAGE promoter was determined using a ChIP assay. As shown in Fig. 4, high glucose conditions increased the binding of this transcription factor to the RAGE promoter. This result suggested that the expression of RAGE expression was regulated by $\mathrm{NF}-\kappa \mathrm{B}$. 


\section{Discussion}

The present study showed the first evidence, to the best of our knowledge, of HMGB1 translocatton into the cytoplasm of retinal pericytes in response to high glucose in vitro and in diabetic conditions in vivo. Through immunofluorescence staining and western blot analyses for HMGB1, the present study determined that HMGB1 translocated from the nucleus to the cytoplasm in response to high glucose. These observations indicated that high glucose was an important regulator of the subcelluar distribution of HMGB1 in the retinal pericytes.

Retinal pericytes wrap around the microvascular endothelium to provide vascular stability and regulate capillary blood flow. The retina has the highest number of pericytes in the body (32), and their loss is considered to be a hallmark of early diabetic retinopathy (33). Pericytes are important in the barrier function of microvessels and assist in regulating inflammatory processes, including the leakage of plasma proteins (34). Diabetes has been shown to be associated with the upregulation of various pro-inflammatory mediators in the retina, including intercellular adhesion molecule 1, vascular endothelial growth factor, NF- $\mathrm{BB}$, inducible nitric oxide synthase and transforming growth factor- $\beta$, and localized inflammatory processes are considered to be involved in the development of diabetic retinopathy $(35,36)$. The data presented in the present study showed that high glucose significantly induced the cytoplasmic expression of the pro-inflammatory mediator, HMGB1, in retinal pericytes, suggesting that increased inflammation is an important contributing factor to the progressive injury of pericytes during the development of diabetic retinopathy.

The present study also showed that the expression of RAGE was markedly enhanced in high glucose-treated retinal pericytes. HMGB1 is a specific ligand for RAGE (30). RAGE is expressed at low levels in a variety of cell types, however, its expression is increased by the cellular activation, which occurs during inflammation $(37,38)$. RAGE was originally identified by its ability to bind advanced glycation end products (AGEs). Increased glucose levels lead to the formation of AGEs. However, the importance of AGEs as ligands of RAGE in vivo remains controversial, as proteins modified by AGEs to the extent necessary to bind to RAGE are unlikely to exist in physiological systems in vivo (39-41). By contrast, the HMGB1 protein is present at sites of inflammation in vivo at concentrations, which activate RAGE (42). RAGE is also found on retinal pericytes (43). This suggests that HMGB1 is a functional mediator, which is involved in the induction of diabetic retinopathy by signaling through these receptors.

The present study also found that high glucose-induced HMGB1 increased the activity of NF- $\mathrm{\kappa B}$. It has been reported that the NF- $\kappa B$ system is a major intracellular signaling pathway downstream of RAGE (44). The NF- $\mathrm{kB}$ transcription factor is a key regulator of inflammation, immune response, cell survival and cell proliferation (45). In the present study, it was found that the transcriptional activity of NF- $\mathrm{KB}$ was significantly enhanced in retinal pericytes by high glucose in vitro and under diabetic conditions in vivo. This is consistent with reports that the constitutive activation of NF- $\mathrm{KB}$ is essential for retinal pericyte damage (46-48). The pro-inflammatory role of NF- $\mathrm{kB}$ has been implicated in pericyte injury through the transcriptional activation of NF- $\mathrm{KB}$-dependent inflammatory mediators. Pro-inflammatory cytokines, including tumor necrosis factor- $\alpha$ and monocyte chemoattractant protein-1, are also regulated by NF-KB in retinal pericytes $(48,49)$. In addition, ChIP analysis showed that high glucose also increased the binding of NF- $\kappa B$ to the RAGE promoter. These results demonstrated that the increased expression of RAGE was a consequence of the HMGB1-induced activation of NF- $\mathrm{kB}$.

In conclusion, the results of the present study indicated that hyperglycemia-induced HMGB1 release may induce retinal pericyte injury under diabetic conditions. It was also demonstrated that the pathogenic role of HMGB1 may be dependent on the NF- $\mathrm{BB}$-dependent regulation of RAGE. Whether the markedly elevated protein levels of HMGB1 in patients with diabetic retinopathy is responsible for the retinal pericyte injury remains to be elucidated. If this is the case, such knowledge may be considered in the design of potential therapeutic strategies for the treatment of diabetic retinopathy.

\section{Acknowledgements}

This study was supported by a grant (grant no. K15270 and K16817) from the Korea Institute of Oriental Medicine.

\section{References}

1. The DCCT Research Group: The effect of intensive treatment of diabetes on the development and progression of long-term complications in insulin-dependent diabetes mellitus. $\mathrm{N}$ Engl J Med 329: 977-986, 1993.

2. Joussen AM, Poulaki V, Le ML, Koizumi K, Esser C, Janicki H, Schraermeyer U, Kociok N, Fauser S, Kirchhof B, et al: A central role for inflammation in the pathogenesis of diabetic retinopathy. FASEB J 18: 1450-1452, 2004.

3. King GL: The role of inflammatory cytokines in diabetes and its complications. J Periodontol 79: 1527-1534, 2008.

4. Muller S, Ronfani L and Bianchi ME: Regulated expression and subcellular localization of HMGB1, a chromatin protein with a cytokine function. J Intern Med 255: 332-343, 2004.

5. Bianchi ME: Significant (re)location: How to use chromatin and/or abundant proteins as messages of life and death. Trends Cell Biol 14: 287-293, 2004.

6. Ulloa L and Messmer D: High-mobility group box 1 (HMGB1) protein: Friend and foe. Cytokine Growth Factor Rev 17: 189-201, 2006.

7. Scaffidi P, Misteli T and Bianchi ME: Release of chromatin protein HMGB1 by necrotic cells triggers inflammation. Nature 418: 191-195, 2002.

8. Bianchi ME and Manfredi A: Chromatin and cell death. Biochim Biophys Acta 1677: 181-186, 2004.

9. Lotze MT and Tracey KJ: High-mobility group box 1 protein (HMGB1): Nuclear weapon in the immune arsenal. Nat Rev Immunol 5: 331-342, 2005.

10. Jiang W, Li J, Gallowitsch-Puerta M, Tracey KJ and Pisetsky DS: The effects of CpG DNA on HMGB1 release by murine macrophage cell lines. J Leukoc Biol 78: 930-936, 2005.

11. Riedemann NC, Guo RF and Ward PA: The enigma of sepsis. J Clin Invest 112: 460-467, 2003.

12. Fiuza C, Bustin M, Talwar S, Tropea M, Gerstenberger E, Shelhamer JH and Suffredini AF: Inflammation-promoting activity of HMGB1 on human microvascular endothelial cells. Blood 101: 2652-2660, 2003.

13. Wang H, Bloom O, Zhang M, Vishnubhakat JM, Ombrellino M, Che J, Frazier A, Yang H, Ivanova S, Borovikova L, et al: HMG-1 as a late mediator of endotoxin lethality in mice. Science 285 : 248-251, 1999.

14. Taniguchi N, Kawahara K, Yone K, Hashiguchi T, Yamakuchi M, Goto M, Inoue K, Yamada S, Ijiri K, Matsunaga S, et al: High mobility group box chromosomal protein 1 plays a role in the pathogenesis of rheumatoid arthritis as a novel cytokine. Arthritis Rheum 48: 971-981, 2003. 
15. Inoue K, Kawahara K, Biswas KK, Ando K, Mitsudo K, Nobuyoshi M and Maruyama I: HMGB1 expression by activated vascular smooth muscle cells in advanced human atherosclerosis plaques. Cardiovasc Pathol 16: 136-143, 2007.

16. Kalinina N, Agrotis A, Antropova Y, DiVitto G, Kanellakis P Kostolias G, Ilyinskaya O, Tararak E and Bobik A: Increased expression of the DNA-binding cytokine HMGB1 in human atherosclerotic lesions: Role of activated macrophages and cytokines. Arterioscler Thromb Vasc Biol 24: 2320-2325, 2004.

17. Yao D and Brownlee M: Hyperglycemia-induced reactive oxygen species increase expression of the receptor for advanced glycation end products (RAGE) and RAGE ligands. Diabetes 59: 249-255, 2010.

18. Lupo G, Motta C, Giurdanella G, Anfuso CD, Alberghina M, Drago F, Salomone S and Bucolo C: Role of phospholipases A2 in diabetic retinopathy: In vitro and in vivo studies. Biochem Pharmacol 86: 1603-1613, 2013.

19. Tamura H, Miyamoto K, Kiryu J, Miyahara S, Katsuta H, Hirose F, Musashi K, Yoshimura N: Intravitreal injection of corticosteroid attenuates leukostasis and vascular leakage in experimental diabetic retina. Invest Ophthalmol Vis Sci 46 1440-1444, 2005.

20. Jonas JB and Söfker A: Intraocular injection of crystalline cortisone as adjunctive treatment of diabetic macular edema. Am J Ophthalmol 132: 425-427, 2001

21. Cai J, Kehoe O, Smith GM, Hykin P and Boulton ME: The angiopoietin/Tie-2 system regulates pericyte survival and recruitment in diabetic retinopathy. Invest Ophthalmol Vis Sci 49: 2163-2171, 2008.

22. Capetandes A and Gerritsen ME: Simplified methods for consistent and selective culture of bovine retinal endothelial cells and pericytes. Invest Ophthalmol Vis Sci 31: 1738-1744, 1990.

23. Kondo T, Hosoya K, Hori S, Tomi M, Ohtsuki S, Takanaga H, Nakashima E, Iizasa H, Asashima T, Ueda M, et al: Establishment of conditionally immortalized rat retinal pericyte cell lines (TR-rPCT) and their application in a co-culture system using retinal capillary endothelial cell line (TR-iBRB2). Cell Struct Funct 28: 145-153, 2003.

24. Wang YL, Hui YN, Guo B and Ma JX: Strengthening tight junctions of retinal microvascular endothelial cells by pericytes under normoxia and hypoxia involving angiopoietin-1 signal way. Eye (Lond) 21: 1501-1510, 2007.

25. Cacicedo JM, Benjachareowong S, Chou E, Ruderman NB and Ido Y: Palmitate-induced apoptosis in cultured bovine retinal pericytes: Roles of NAD (P)H oxidase, oxidant stress, and ceramide. Diabetes 54: 1838-1845, 2005.

26. Schreiber E, Matthias P, Müller MM and Schaffner W: Rapid detection of octamer binding proteins with 'mini-extracts', prepared from a small number of cells. Nucleic Acids Res 17: 6419, 1989

27. Bradford MM: A rapid and sensitive method for the quantitation of microgram quantities of protein utilizing the principle of protein-dye binding. Anal Biochem 72: 248-254, 1976.

28. von Knethen A, Callsen D and Brüne B: NF-kappaB and AP-1 activation by nitric oxide attenuated apoptotic cell death in RAW 264.7 macrophages. Mol Biol Cell 10: 361-372, 1999.

29. Vos MD, Ellis CA, Elam C, Ulku AS, Taylor BJ and Clark GJ RASSF2 is a novel K-Ras-specific effector and potential tumor suppressor. J Biol Chem 278: 28045-28051, 2003.

30. Hori O, Brett J, Slattery T, Cao R, Zhang J, Chen JX, Nagashima M, Lundh ER, Vijay S, Nitecki D, et al: The receptor for advanced glycation end products (RAGE) is a cellular binding site for amphoterin. Mediation of neurite outgrowth and co-expression of rage and amphoterin in the developing nervous system. J Biol Chem 270: 25752-25761, 1995.
31. Clynes R, Moser B, Yan SF, Ramasamy R, Herold K and Schmidt AM: Receptor for AGE (RAGE): Weaving tangled webs within the inflammatory response. Curr Mol Med 7: 743-751, 2007.

32. Motiejunaite R and Kazlauskas A: Pericytes and ocular diseases. Exp Eye Res 86: 171-177, 2008

33. Kuwabara T and Cogan DG: Retinal vascular patterns. VII. Acellular change. Invest Ophthalmol 4: 1049-1064, 1965.

34. Sims DE: Diversity within pericytes. Clin Exp Pharmacol Physiol 27: 842-846, 2000.

35. Joussen AM, Murata T, Tsujikawa A, Kirchhof B, Bursell SE and Adamis AP: Leukocyte-mediated endothelial cell i njury and death in the diabetic retina. Am J Pathol 158: 147-152, 2001.

36. Kern TS: Contributions of inflammatory processes to the development of the early stages of diabetic retinopathy. Exp Diabetes Res 2007: 95103, 2007.

37. Hofmann MA, Drury S, Fu C, Qu W, Taguchi A, Lu Y, Avila C, Kambham N, Bierhaus A, Nawroth P, et al: RAGE mediates a novel proinflammatory axis: A central cell surface receptor for S100/calgranulin polypeptides. Cell 97: 889-901, 1999.

38. Bierhaus A, Schiekofer S, Schwaninger M, Andrassy M, Humpert PM, Chen J, Hong M, Luther T, Henle T, Klöting I, et al: Diabetes-associated sustained activation of the transcription factor nuclear factor-kappaB. Diabetes 50: 2792-2808, 2001.

39. Thornalley PJ: Dietary AGEs and ALEs and risk to human health by their interaction with the receptor for advanced glycation endproducts (RAGE)-an introduction. Mol Nutr Food Res 51: 1107-1110, 2007.

40. Ramasamy R, Yan SF and Schmidt AM: Arguing for the motion: Yes, RAGE is a receptor for advanced glycation endproducts. Mol Nutr Food Res 51: 1111-1115, 2007.

41. Heizmann CW: The mechanism by which dietary AGEs are a risk to human health is via their interaction with RAGE: Arguing against the motion. Mol Nutr Food Res 51: 1116-1119, 2007.

42. Foell D, Wittkowski H, Vogl T and Roth J: S100 proteins expressed in phagocytes: A novel group of damage-associated molecular pattern molecules. J Leukoc Biol 81: 28-37, 2007.

43. Yamagishi S, Takeuchi M, Matsui T, Nakamura K, Imaizumi T and Inoue H: Angiotensin II augments advanced glycation end product-induced pericyte apoptosis through RAGE overexpression. FEBS Lett 579: 4265-4270, 2005.

44. Yan SD, Schmidt AM, Anderson GM, Zhang J, Brett J, Zou YS, Pinsky D and Stern D: Enhanced cellular oxidant stress by the interaction of advanced glycation end products with their receptors/binding proteins. J Biol Chem 269: 9889-9897, 1994.

45. Karin M and Ben-Neriah Y: Phosphorylation meets ubiquitination: The control of NF-[kappa]B activity. Annu Rev Immunol 18: 621-663, 2000.

46. Kim J, Kim OS, Kim CS, Kim NH and Kim JS: Cytotoxic role of methylglyoxal in rat retinal pericytes: Involvement of a nuclear factor-kappaB and inducible nitric oxide synthase pathway. Chem Biol Interact 188: 86-93, 2010.

47. Kim J, Son JW, Lee JA, Oh YS and Shinn SH: Methylglyoxal induces apoptosis mediated by reactive oxygen species in bovine retinal pericytes. J Korean Med Sci 19: 95-100, 2004.

48. Romeo G, Liu WH, Asnaghi V, Kern TS and Lorenzi M: Activation of nuclear factor-kappaB induced by diabetes and high glucose regulates a proapoptotic program in retinal pericytes. Diabetes 51: 2241-2248, 2002.

49. Zhang SX, Wang JJ, Dashti A, Wilson K, Zou MH, Szweda L, Ma JX and Lyons TJ: Pigment epithelium-derived factor mitigates inflammation and oxidative stress in retinal pericytes exposed to oxidized low-density lipoprotein. J Mol Endocrinol 41: 135-143, 2008 . 